\title{
Référentiels aspecto-temporels : une approche formelle et cognitive appliquée au français
}

\author{
Jean-Pierre Desclés \\ LaLIC, Université de Paris-Sorbonne \\ jean-pierre.descles@paris-sorbonne.fr \\ Zlatka Guentchéva \\ CNRS, France \\ guentche@vjf.cnrs.fr
}

\section{Introduction}

La conceptualisation de la temporalité conçue comme un axe linéaire dont le «maintenant» serait une coupure entre «le passé » déjà réalisé et "le futur» encore à venir est certainement un obstacle épistémologique (au sens de G. Bachelard) pour décrire, structurer et finalement comprendre la temporalité, qui est organisée cognitivement par les langues, et que la linguistique doit reconstruire à partir d'une analyse sémantique des expressions temporelles et aspectuelles. L'observation des données du français (l'exercice peut être appliqué à d'autres langues) met bien en évidence la non-linéarité d'une temporalité catégorisée à partir des marqueurs grammaticalisés. Plus généralement, la temporalité ne peut pas être décrite dans un seul référentiel temporel homogène et symétrique autour du «maintenant de l'énonciation ». Il s'agit donc de «penser la temporalité linguistique » d'une façon plus complexe.

Dans l'analyse sémantique des expressions de la temporalité linguistique, il faut prendre en compte d'un côté, les relations purement temporelles, c'est-à-dire la concomitance par indentification $(=)$, et la différenciation temporelle $(\neq)$ (d'antériorité et de postériorité), en particulier pour repérer les situations verbalisées par rapport à la situation d'énonciation, et de l'autre côté, les trois opérateurs aspectuels fondamentaux qui portent globalement sur une relation prédicative ${ }^{1}$ produisant une différenciation entre « état» (ce qui est stable ou stabilisé), " événement» (transition entre deux états) et "processus » (changement en cours, donc inaccompli). ${ }^{2}$ Outre ces trois notions aspectuelles et les relations temporelles d'identification et de différenciation, il faudrait distinguer différents types de référentiels temporels, en particulier celui qui est organisé par l'acte énonciatif, afin de construire un référentiel énonciatif distinct du référentiel externe. E. Benveniste (1966) a insisté sur la différence entre, d'un côté, le «temps chronique ( (nous pourrions ajouter le «temps calendaire »), le «temps physique » et, de l'autre côté, le « temps linguistique »:
«Autre chose est de situer un événement dans le temps chronique, autre chose de l'insérer dans le temps de la langue. C'est par la langue que se manifeste l'expérience humaine du temps et le temps linguistique nous apparaît également irréductible au temps chronique et au temps physique »

(Benveniste, 1974 : 73).

Il est également nécessaire d'introduire des référentiels non actualisés dans lesquelles les situations verbalisées ne sont plus repérables par rapport à leur énonciation. Ainsi, outre le référentiel énonciatif, le référentiel externe (le monde externe) et le référentiel non actualisé, d'autres types de référentiels temporels se révèlent également nécessaires pour une analyse sémantique de certains énoncés de français (et de la plupart des langues) : le référentiel des discours rapportés REN2, le référentiel des cadres de pensées RCP, le référentiel des commentaires RCOM, le référentiel des vérités générales RVG, le référentiel des situations possibles RPOS, le référentiel des savoirs partagés RSP... Dans cet article, nous nous proposons d'expliciter ce qu'il faut entendre par «référentiel temporel» et par « système de référentiels » et de les illustrer avec des exemples de ces différents référentiels. 


\section{Notion de référentiel}

La notion de référentiel, en particulier celle de référentiel temporel, est essentielle dans l'activité scientifique, que ce soit en physique ou dans d'autres domaines de la connaissance (psychologie cognitive, entre autres...). Les langues sont des systèmes de représentations sémiotiques qui sont capables de représenter non seulement les situations actuelles directement perçues, mais aussi des situations passées, visées, imaginées, possibles, contrefactuelles...; les différents mécanismes de référenciation temporelle sont donc l'un des domaines d'étude les plus essentiels de la linguistique cognitive. Pour rendre compte de ces mécanismes, les recherches épistémologiques sur la physique ${ }^{3}$ nous ont appris à distinguer explicitement ce qui est observé et ce qui est référencé. Les représentations doivent donc dégager différents référentiels, en particulier, le référentiel de l'observateur et celui des faits décrits. La réflexion de Galilée a montré qu'un référentiel peut être en mouvement par rapport à un autre référentiel ; que la description d'un mouvement, ou d'un changement, dépend étroitement du référentiel choisi ; que ce qui est fixe dans un référentiel peut être en mouvement dans un autre référentiel... Ces acquis de la pensée scientifique et philosophique doivent trouver un écho dans l'analyse des langues. En nous situant dans une approche énonciative, l'énonciateur devient comparable à un observateur qui doit, par son énonciation même, organiser ce qu'il observe et énonce, dans un référentiel qui le contient et qui s'est détaché des référentiels externes (temps physique, temps cosmique, temps calendaire, temps des chroniques et temps des annales...). Cette capacité à se détacher du monde externe est l'une des capacités les plus profondes de l'homme et elle se reflète dans son langage; elle est à l'origine des catégories de la personne et des déictiques spatiaux et temporels. Que le temps avance ou que l'énonciateur soit en mouvement dans la temporalité externe n'intervient pas dans le processus d'énonciation car, en énonçant, l'énonciateur crée son propre référentiel avec, dans ce référentiel, l'introduction d'un repère fixe: le repère ' $\mathrm{T}^{0}$ '. Ce dernier, lorsqu'il se projette dans le référentiel externe, devient un repère mobile ' $\mathrm{t}_{\mathrm{m}}$ '. La capacité à se détacher du monde externe entraîne une rupture entre le référentiel énonciatif et les référentiels externes, chacun des référentiels ayant sa propre organisation temporelle. Sans cette rupture aucun dialogue réussi entre un énonciateur et son co-énonciateur ne pourrait avoir lieu, puisque le temps, en fuyant, déplacerait sans cesse tout repère temporel stable de référence, rendant ainsi impossible tout ajustement inter-énonciateurs, car ce qui n'était encore que futur deviendra passé, et ce qui est présent ne sera plus présent... Dans ses Confessions (Livre XI), Saint Augustin ne distinguait pas le référentiel de l'énonciation de la référence externe, les deux référentiels étant, dans son analyse, confondus :

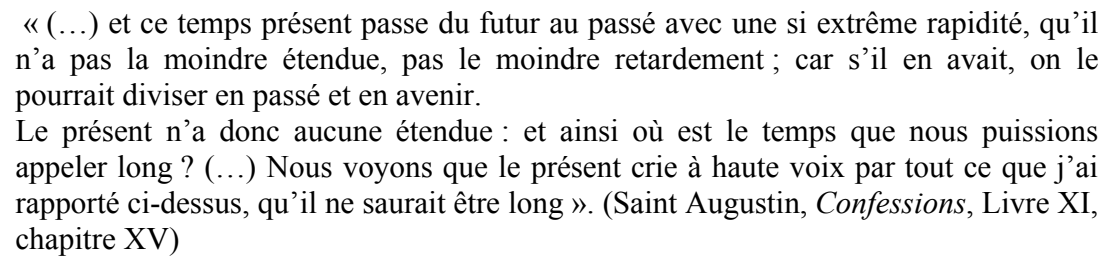

Si Augustin a su analyser magistralement la notion phénoménale du «temps présent », « sans étendue », il n'a pas analysé pour autant la sémantique des temps grammaticaux mais ce n'était pas, après tout, son objet d'étude. L'analyse des temps grammaticaux fait apparaître vite que la notion de «présent » ne peut pas être réduite à un instant fuyant et ponctuel. Appréhender « le présent du langage » dans le référentiel énonciatif créé par l'acte d'énonciation évite de confondre le référentiel énonciatif avec le référentiel externe. Beaucoup de modèles linguistiques de la temporalité ne sont pas toujours très clairs sur cette distinction essentielle à nos yeux. Par exemple, H. Reichenbach $(1947)^{4}$, repris très souvent par des linguistes ${ }^{5}$, pose un instant ponctuel ' $\mathrm{T}^{0}$, d'énonciation (considéré comme « le moment d'énonciation ») sans préciser si cet instant est situé dans un référentiel externe, - ce serait alors un instant en mouvement -, ou dans un autre référentiel - ce qui en ferait un repère fixe pour les autres repères, le repère de l'événement et le repère du point de vue. Dans une approche de la temporalité linguistique, l'instant ' $\mathrm{T}^{0}$, ne doit pas être considéré comme le «moment d'énonciation » puisqu'énoncer consomme du temps (moi, énonciateur, je suis en train de dire...). L'énonciation est donc un processus qui se déroule dans le temps au sein du référentiel énonciatif. Le processus d'énonciation est fondamentalement inaccompli, car il se réalise durant un intervalle ' $\mathrm{J}_{0}$ ' d'instants, avec un nécessaire premier instant de 
l'énonciation - donc avec une borne fermée à gauche $\gamma\left(\mathrm{J}_{0}\right)$ de l'intervalle ' $\mathrm{J}_{0}$ '-, et sans dernier instant donc avec une borne ouverte à droite $\delta\left(\mathrm{J}_{0}\right)$. En effet, si le processus était accompli, il y aurait un dernier instant d'énonciation, et l'acte d'énonciation deviendrait alors un événement relégué dans le passé ; elle ne serait donc plus à la source du «présent linguistique ». La borne droite $\delta\left(\mathrm{J}_{0}\right)$ constitue le repère fixe ' $\mathrm{T}^{0}$ ' du le référentiel énonciatif : $\left[\mathrm{T}^{0}=\delta\left(\mathrm{J}_{0}\right)\right]$; ce repère est exclu de l'intervalle d'énonciation ' $\mathrm{J}_{0}$ ', il en est simplement l'une des bornes.

Un système de repérage, inspiré par A. Culioli, est construit à l'aide d'un archirelateur REP de repérage, dont les valeurs sont l'identification (dans le domaine de la temporalité : la concomitance), la différenciation (antériorité / postériorité) et la rupture. Une rupture est une relation externe de repérage entre deux référentiels ; les relations d'indentification ou de différenciation étant des relations internes de repérage à l'intérieur d'un même référentiel.

Donnons maintenant la définition générale d'un référentiel temporel et celle d'un système de référentiels temporels :

- Un référentiel temporel est un système de repérage, constitué par un ensemble continu et ordonné d'instants : chaque instant t peut être repéré par rapport à un autre instant $\mathrm{t}^{\prime}$, soit par concomitance $(=)$, soit par différenciation $(\neq)$-antériorité ou postériorité. L'ensemble étant continu, on peut donc y définir des intervalles topologiques d'instants, avec des bornes ouvertes (non prise en compte de premiers ou de derniers instants dans l'intervalle) et fermées (prise en compte de premiers ou de derniers instants dans l'intervalle).

- Un système de référentiels temporels est structuré par une relation de rupture, notée \#, (supposée irréflexive, transitive et symétrique), entre deux référentiels ; les référentiels temporels mis en rupture ont des organisations temporelles autonomes. Plus précisément, la relation de rupture tient entre deux référentiels $\mathbf{R}_{\mathbf{i}}$ et $\mathbf{R}_{\mathbf{j}}$ lorsque pour tout instant $\mathrm{t}$ d'un référentiel temporel $\mathbf{R}_{\mathbf{i}}$, il existe un instant $\mathrm{u}$ du référentiel $\mathbf{R}_{\mathbf{j}}$ tel que : (i) u n'est pas repérable, par identification $(=)$ ou différenciation $(\neq)$, dans le référentiel $\mathbf{R}_{\mathbf{i}}-\mathrm{u}$ entretient une relation de rupture avec $\mathrm{t}$ et nous notons [u \# $\mathrm{t}$ ] ; (ii) pour tout instant $\mathrm{v}$ repérable par rapport à u dans le référentiel $\mathbf{R}_{\mathbf{j}}$, l'instant $\mathrm{t}$ est également en rupture avec $\mathrm{v}([\mathrm{v} \# \mathrm{t}])$. Deux référentiels en rupture, l'un par rapport à l'autre, peuvent être par ailleurs éventuellement synchronisés entre eux, à condition toutefois de spécifier explicitement ce synchronisme entre deux instants de chacun des référentiels. Il existe plusieurs formes de systèmes de référentiels (Desclés, 1980) : spirale, came...

\section{Une théorie aspectuelle : un bref rappel}

La temporalité du langage ne peut pas être décrite sans tenir compte de l'aspectualité. Toute notion aspectuelle implique une temporalité sous-jacente, la plupart des relations temporelles entre situations exprimées fait appel à des aspectualisations de celles-ci et donc à des relations entre bornes ouvertes ou fermées. Rappelons sommairement la théorie aspectuelle des états, événements et processus (par exemple, Lyons 1970 ; Comrie 1976 ; Mourelatos 1978) que nous avons déjà exposée dans diverses publications (Desclés 1980, 2004; Guentchéva 1990, Desclés \& Guentchéva 2000...). Pour un panorama des différentes valeurs sémantiques des temps du français, on peut se rapporter à l'ouvrage de $\mathrm{Ch}$. Touratier (1996).

Chaque relation prédicative élémentaire est constituée par une opération de prédication et, éventuellement, par des opérations supplémentaires de diathèse (orientation de la relation prédicative) et de thématisation /topicalisation; elle met en jeu un prédicat verbal appliqué à divers arguments, chaque argument ayant un rôle grammatical spécifique (agent, patient, localisateur, localisé, destinataire...). Cette relation prédicative devient aspectualisée, appelée procès, lorsqu'elle est appréhendée par l'énonciateur sous la forme soit d'un état (c'est-à-dire comme une relation statique où aucun changement n'est pris en compte), soit d'un événement (qui exprime une transition entre un état antérieur et un état postérieur), soit encore d'un processus en cours (qui exprime un changement ou une évolution affectant l'un des arguments de la relation prédicative). Ces trois notions aspectuelles ne sont pas indépendantes. En effet : 
- Un état (non permanent) est borné par deux événements : un événement qui fait entrer dans l'état; un événement qui en fait sortir.

- Un événement établit une transition entre un état antérieur (avant) et un état postérieur (après) ; il comporte donc un début et une fin.

- Un processus (inaccompli) exprime une évolution saisie dans son développement; il implique un premier instant mais pas la prise en compte d'un dernier instant de réalisation (qui est alors un terme d'accomplissement) ; lorsque le processus devient accompli (donc avec un dernier instant), il engendre un événement.

Il existe une typologie des états, des événements et des processus (nous renvoyons sur ce point à nos publications antérieures).

Donnons quelques exemples de procès associés à la même relation prédicative («Luc fumer unecigarette ») avec les trois énoncés :
(a) Luc a fumé une cigarette, cet après-midi (événement passé)
(b) Luc fume (en ce moment) une cigarette (processus)
(c) Luc a enfin fumé une cigarette : il est devenu grand!

(état résultant ou état acquis par le sujet)

L'analyse aspecto-temporelle d'un procès implique des intervalles de réalisation (ou intervalles de validation), c'est-à-dire des intervalles où, pour chaque instant de l'intervalle, la relation prédicative est vraie. Ces intervalles ont des propriétés topologiques différentes selon que les bornes (premier ou dernier instant de réalisation) sont prises ou non en compte. Un événement implique la prise en compte d'un début et d'une fin ; il est donc réalisé, en tant que transition, sur un intervalle fermé. Un état exclut la prise en compte d'une borne de début et d'une borne de fin, il est réalisé sur un intervalle ouvert ; même si de telles bornes existent (par exemple dans un état contingent), elles n'appartiennent pas à l'intervalle de réalisation de l'état car elles appartiennent soit à l'événement antérieur qui a conduit à l'état, soit à l'événement postérieur qui en fait sortir. Le processus inaccompli implique nécessairement une discontinuité initiale du changement entre l'antérieur stable précédent et le changement lui-même; étant inaccompli, il n'implique pas la prise en compte d'une borne finale car, si c'était le cas, le processus deviendrait alors un événement et son évolution serait accomplie.

L'énonciation est un acte de prise en charge d'un procès. En tant qu'acte énonciatif ( « Je suis en train de dire : (...)»), il est un processus inaccompli sans dernier instant de réalisation; il se réalise sur un intervalle (non ponctuel ' $\mathrm{J}_{0}$ ' dont la borne de droite est ouverte et notée ' $\mathrm{T}$ '.

Pour acquérir le statut d'un énoncé, un procès doit être pris en charge par un énonciateur et donc inséré dans un référentiel : soit dans le référentiel énonciatif de l'énonciateur, en étant repéré par une relation temporelle par rapport au processus énonciatif, soit dans un autre référentiel lui-même repéré, au sens large, par rapport au référentiel énonciatif.

\section{Référentiel énonciatif : REN}

Dans le référentiel créé par l'énonciateur, appelé référentiel énonciatif, et désigné par le REN, les relations prédicatives qui s'y inscrivent sont aspectualisées, d'où l'opérateur aspectuel $\mathrm{ASP}_{\mathbf{I}}$ (où $\mathrm{ASP}_{\mathbf{I}}$ prend les valeurs ETAT, EVEN, ou PROC) portant sur la relation prédicative qui se réalise sur un intervalle topologique I; la relation aspectualisée doit être, par ailleurs, repérée de façon interne, au moyen du relateur REP, par rapport au processus énonciatif, désigné par JE-DIS, lui-même aspectualisé sous la forme d'un processus qui se réalise sur l'intervalle d'énonciation $\mathrm{J}_{0}$, d'où l'opérateur $\mathrm{PROC}_{\mathrm{J} 0}$. Nous obtenons ainsi le schème énonciatif aspectualisé:

$$
\text { PROC }_{\mathbf{J} 0}\left(\mathrm{JE}-\mathrm{DIS}\left(\left[\mathrm{ASP}_{\mathrm{I}}\left(\text { relation prédicative) } \&\left[\delta(\mathrm{I}) \underline{\mathrm{REP}} \delta\left(\mathrm{J}_{0}\right)\right]\right]\right)\right)\right.
$$


Les symboles ' $\delta(\mathrm{I})$ ' et ' $\delta\left(\mathrm{J}_{0}\right)$ ' désignent les bornes droites des intervalles respectifs 'I' et ' $\mathrm{J}_{0}$ ' qui sont repérés entre eux de façon interne par REP. La relation temporelle $\left[\delta(\mathrm{I}) \underline{\mathrm{REP}} \delta\left(\mathrm{J}_{0}\right)\right]$ constitue les « coordonnées énonciatives » du procès. Le référentiel REN a une structure non symétrique où le repère fixe $\mathrm{T}^{0}$ introduit une coupure continue ${ }^{6}$ entre : (i) l'ensemble totalement ordonné des instants réalisés, d'où le domaine modal du certain réalisé et (ii) l'ensemble ordonné, mais non linéairement (en termes mathématiques, c'est un demi-treillis) des instants non encore réalisés et des instants visés dans un futur possible, constituant ainsi le domaine modal non-certain du non-réalisé. L'instant ' $\mathrm{T}^{0}$ ' est fixe dans REN (c'est la borne droite ouverte de l'intervalle ' $\mathrm{J}_{0}$ ' de réalisation du processus énonciatif, d'où $\left[\delta\left(\mathrm{J}_{0}\right)=\mathrm{T}^{0}\right]$ ) ; il constitue «le premier instant du non-réalisé », sa projection sur un référentiel externe, le temps physique par exemple, est un instant $\mathrm{t}_{\mathrm{m}}$ qui, lui, change avec le flux du temps.

Les instants déjà réalisés sont ordonnés par une réminiscence mémorielle ou, inversement, par une organisation chronologique, selon que l'on remonte dans le passé (réalisé) en s'éloignant de ' $\mathrm{T}^{0}$, , ou que l'on se rapproche de ' $\mathrm{T}^{0}$ ' en suivant fidèlement l'ordre de la successivité des événements. L'ensemble des instants non réalisés n'a pas la même structure que celle du domaine réalisé. En effet, ce qui va arriver ou ce qui arrive reste non-certain, alors que ce qui est déjà arrivé est devenu certain, ou présenté comme tel à un co-énonciateur par un acte d'énonciation. Les notions aspecto-temporelles de réalisé / non réalisé entrent en interaction avec les notions modales du certain / non-certain, comme l'ont remarqué un grand nombre d'études qui ont dégagé la notion de TAM (Temps-Aspects-Modalités). Le domaine réalisécertain dans le référentiel énonciatif REN ne doit pas être confondu avec le réalisé dans le référentiel externe REX puisque le REN présente la vision et le point de vue de l'énonciateur dans son référentiel et que ce dernier peut entrer en contradiction avec ce qui a été effectivement réalisé dans le référentiel externe : l'énonciateur peut se tromper, mentir volontairement ou involontairement...

Tous les événements futurs sont possibles ou probables mais jamais certains. En reprenant un exemple célèbre d'Aristote Demain, il y aura une bataille navale, on ne peut pas considérer que l'événement visé ait nécessairement une occurrence, seule sa probabilité est présentée ; en revanche, en énonçant Il y a eu, hier, une bataille navale, on indique une occurrence certaine d'un événement réalisé. Le non-réalisé est ainsi organisé selon une organisation arborescente ${ }^{7}$ où, dans chaque branche temporelle, la réalisation de l'occurrence d'un événement visé à partir de l'acte d'énonciation peut être considérée comme possible, ce qui veut dire que l'absence d'occurrence de sa réalisation reste également possible et doit s'inscrire dans une branche alternative. La réalisation d'une occurrence possible (envisageable) d'un événement peut être présentée comme étant probable et, dans certains cas, même présentée comme étant quasi-certaine, ce qui signifie que l'absence de son occurrence doit être également envisagée comme restant seulement possible ou avec un degré de probabilité beaucoup plus faible. 


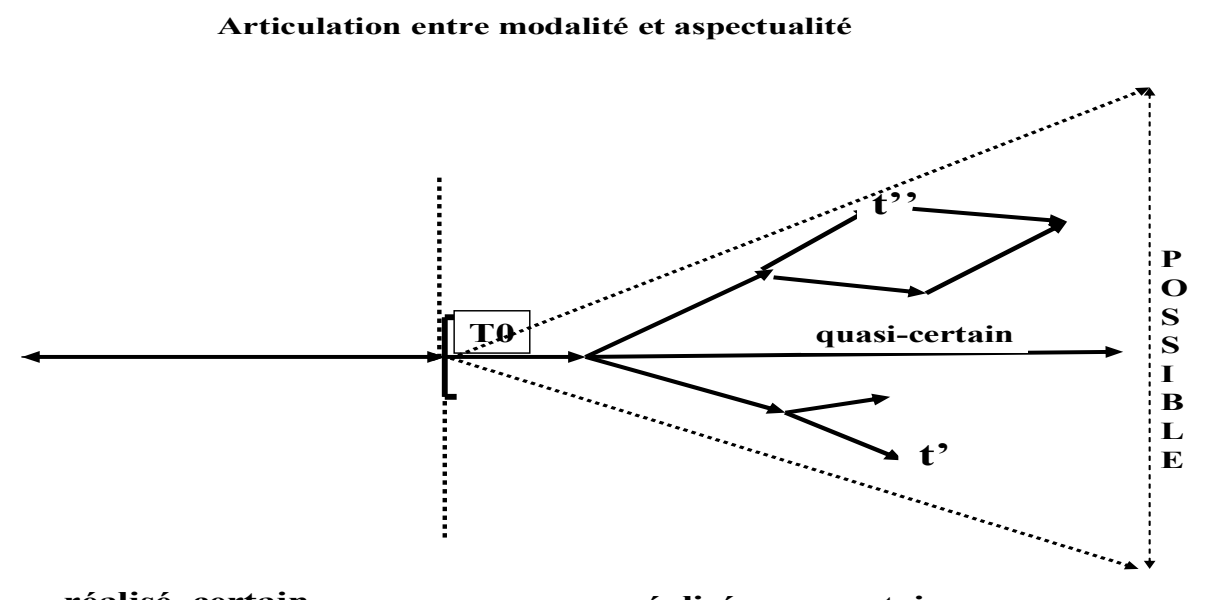

Figure 1 : Non-symétrie dans le référentiel énonciatif

Comparons les exemples suivants :
(a) Luc part (est en train de partir)
(b) Luc part tous les étés en vacances
(c) Luc part à l'instant (de mon bureau)
(d) Luc part demain
(e) Luc va partir demain
(f) Luc partira demain
(g) Il se peut que Luc parte demain

L'énoncé (3a) exprime un processus en cours; l'énoncé (3b) exprime une habitude, c'est-à-dire une succession d'occurrences d'un même événement, le présent indiquant que la série est ouverte au sens suivant : il n'y a pas de dernière occurrence de l'événement. Ainsi, le processus peut être continu (3a) ou discret (3b), mais dans les deux cas, il y a identification de la borne droite avec la borne ' $\mathrm{T}^{0}$, . Dans (3c), il ne s'agit pas d'un «présent-passé » ou d'un «passé récent ${ }^{8}$ mais d'un processus dont le terme d'accomplissement est indiqué, contrairement à (3a) qui exprime un processus en cours sans que le terme d'accomplissement soit évoqué. Dans (3d), le processus est déjà enclenché : le départ est déjà, dans l'intention de l'énonciateur, en train de se produire, la réalisation complète, c'est-à-dire l'accomplissement du processus, est quasi-certaine et atteinte le lendemain. L'énoncé (3e) exprime une visée présente - le verbe aller est au présent - d'un événement considéré comme quasi-certain (la probabilité de son absence d'occurrence est pratiquement nulle); il ne s'agit donc pas d'un "futur proche », comme cela est souvent avancé dans les grammaires, puisque, dans de nombreux cas, il est difficile de parler de proche (Les américains vont débarquer sur Mars dans dix ans; Le soleil va disparaître dans un milliard d'années); nous avons l'énonciation d'une visée exprimée par un mouvement orienté vers un événement quasi-certain. L'énoncé (3f) introduit une certaine valeur de probabilité : l'événement « Luc partir demain » est présenté comme étant beaucoup plus probable que son absence d'occurrence, bien que cette dernière reste encore possible. Quant à l'énoncé (3g), il introduit une possibilité : l'événement « Luc partir demain » est possible, mais son absence d'occurrence également. 
Le présent construit par chaque acte d'énonciation prend toute sa dimension, y compris sa durée, dans le référentiel énonciatif REN, ce qui n'est pas le cas dans le référentiel externe où le « présent » est fuyant et sans étendue. C'est le processus énonciatif qui fonde et organise la notion langagière de présent. Ce processus consomme du temps et ne peut être réduit à un instant ponctuel qui séparerait le futur à venir et non réalisé du passé réalisé. Est présent, donc inaccompli, tout ce qui est concomitant avec le processus énonciatif, donc toute situation qui, en se déployant dans sa temporalité sous-jacente, est bornée à droite par une borne d'inaccomplissement identifiée à $\mathrm{T}^{0}$. Le présent notionnel (à ne pas confondre avec le temps grammatical du présent) est nécessairement un inaccompli dans le référentiel REN. Une situation, qu'elle soit statique (La mer est belle / Le soleil est levé / Le sable est chand ...) ou évolutive (Luc écrit sa lettre pour le percepteur / Le soleil se lève / La mer devient houleuse / Le sable devient de plus en plus chaud ...), a donc une valeur aspectuelle d'état ou de processus; ces deux valeurs sont représentées à l'aide d'opérateurs aspectuels (d'état ou de processus) qui indiquent que les relations prédicatives qui en sont les opérandes se réalisent sur des intervalles (d'instants) - des intervalles ouverts dans le cas des états, des intervalles fermés à gauche et ouverts à droite, dans le cas des processus. Ces deux types d'intervalles ont en commun une borne droite ouverte concomitante à la borne ' $\mathrm{T}^{0}$ ' du processus énonciatif. Cette borne, exclue de l'intervalle de réalisation, indique l'inaccomplissement du processus ou de l'état puisqu'elle n'est pas un dernier instant de réalisation; son rôle ne peut être compris que dans le référentiel REN. On comprend ainsi que tout événement aspectuel ne peut pas être un inaccompli et ne peut donc pas être un présent. En effet, tout événement, conçu globalement comme une transition, ne peut être inscrit que dans un réalisé passé ou être représenté comme un événement possible à venir9. Le référentiel REN est créé par tout acte de langage qui se détache de toute référence externe, tout en restant compatible avec elle, pour construire un «présent énonciatif» à partir duquel s'organisent différentes valeurs aspecto-temporelles des temps grammaticaux.

Le temps grammatical du présent sert à exprimer ce présent inaccompli mais, comme nous le verrons plus loin, il n'est pas réduit à cette seule fonction. De plus, la valeur aspectuelle d'inaccompli n'est pas seulement concomitante à la valeur inaccomplie du processus énonciatif. Prenons quelques exemples simples construits sur la même relation prédicative "Luc écrire une-lettre » pour illustrer les relations de différenciation dans le réalisé du référentiel REN :

\section{(a) Luc écrit en ce moment une lettre}

(b) Luc écrivait une lettre à son percepteur quand je lui ai téléphoné

(c) Luc a écrit hier matin une lettre au percepteur

(d) Enfin, Luc a écrit sa lettre au percepteur, tu peux maintenant l'envoyer

Les valeurs aspecto-temporelles sont respectivement celles : d'un processus inaccompli concomitant à l'énonciation en (4a); d'un processus inaccompli non concomitant à l'énonciation en (4b) ; d'un événement non concomitant à l'énonciation en $(4 \mathrm{c})$; de l'état résultant d'un événement, cet état étant concomitant à l'énonciation en (4d). Dans (4b), l'événement exprimé par je lui ai téléphoné est sécant au processus en cours Luc écrivait une lettre; le processus est situé dans le passé (par rapport à l'acte d'énonciation) et inaccompli par rapport à l'événement qui lui est sécant; le début de cet événement est donc la borne d'inaccomplissement du processus, elle est exclue de l'intervalle de réalisation du processus mais fait partie de la transition événementielle. Dans (4d), l'état résultant se réalise sur un intervalle ouvert ' $\mathrm{O}$ ', contigu et postérieur à l'intervalle ' $\mathrm{F}$ ' de réalisation de l'événement qui lui a donné naissance ; ce sont les éléments contextuels (enfin / maintenant, tu peux ...) qui permettent de distinguer la valeur d'événement en (4c) et celle d'état résultant en (4d), associées à la même forme du passé composé français (Luc a écrit sa lettre hier matin). L'état résultant peut être concomitant à un autre état ou à un autre processus ; c'est pourquoi nous pouvons parler d'un " accompli présent », exprimée dans d'autres langues par un parfait (un «present perfect» en anglais) ${ }^{10}$. Nous avons donc les représentations métalinguistiques suivantes ${ }^{11}$ :

$$
\text { PROC }_{\mathbf{J} 0}\left(\mathrm{JE}-\mathrm{DIS}\left(\left[\mathrm{PROC}_{\mathbf{J}}(\text { écrire }(\text { Luc, lettre }))\right) \&\left[\delta(\mathrm{J})=\delta\left(\mathrm{J}_{0}\right)\right]\right]\right)
$$


(c') $\quad$ PROC $_{J 0}\left(\right.$ JE-DIS $\left(\left[\operatorname{EVEN}_{\mathrm{F}}(\right.\right.$ écrire $($ Luc, lettre $\left.\left.\left.))\right) \&\left[\delta(\mathrm{F})<\delta\left(\mathrm{J}_{0}\right)\right]\right]\right)$

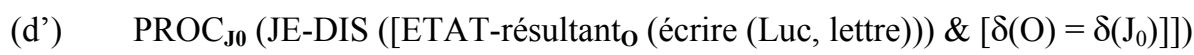

Nous pouvons visualiser ces analyses à l'aide de diagrammes temporels (figure 2) :
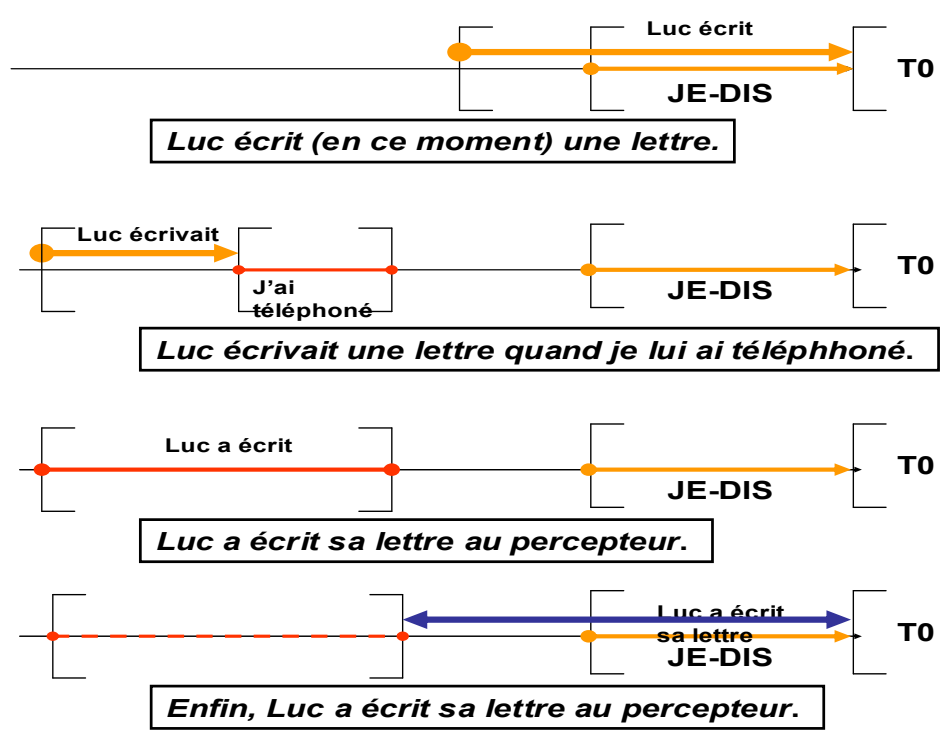

Figure 2 : Visualisation par des diagrammes temporels des énoncés de (4)

Remarquons au passage que l'imparfait n'est pas toujours un temps du "passé », comme on le voit souvent affirmer dans les grammaires. Prenons par exemple les énoncés (5a) et (5b) qui renvoient à des situations «présentes » (dans le référentiel externe REX) ou l'énoncé (5c) qui, lui, porte sur une situation référentielle à venir :
(a) Alors, la petite dame, qu'est-ce qu'elle prenait?
(b) Monsieur, j'étais venu pour vous demander une augmentation
(c) Qu'est-ce qu'on mangeait déjà demain?

Affirmer que l'imparfait « introduit une certaine distanciation », ce qui est le cas des énoncés de (5), n'est pas erroné mais il faut alors préciser la nature de cette distance. Dans (5a), un imparfait appelé 'du marché' (Cohen 1966 : p. 67-68) ou 'forain' (Wilmet 1983: 159), le commerçant décale le procès dans le REN par rapport à son énonciation présente pour indiquer à sa cliente qu'il a bien observé que cette dernière cherchait quelque chose ou attendait à être servie depuis quelque temps. ${ }^{12}$ Dans $(5 b)$, avec un imparfait de politesse, l'énonciateur décale, toujours dans le REN, le procès en laissant à son interlocuteur la capacité de rendre le procès accompli, ce qui était simplement présenté par l'énonciateur comme un processus encore inaccompli, et donc d'en faire un événement du passé, d'où l'effet de politesse. Dans (5c), l'énonciateur introduit implicitement une énonciation rapportée dans le passé (quelqu'un a dit: on mangera plus tard ....). Dans ces trois exemples, nous avons en fait des « situations présentes » dans le référentiel externe REX mais le décalage introduit a lieu dans le référentiel REN ; en confondant les référentiels REN et REX, il semble bien difficile d'expliquer la cohérence des emplois 
(qui peuvent faire référence, comme dans les exemples (5), à des situations présentes ou à-venir) de l'imparfait et espérer dégager un éventuel invariant de l'imparfait ${ }^{13}$. Du reste, devant la multiplicité des valeurs de l'imparfait en français, certains linguistes considèrent qu'il est impossible de lui associer une valeur sémantique invariante dont toutes les autres valeurs en seraient des spécifications contextuelles.

Les intervalles de réalisation des processus identifiés dans un texte n'appartiennent pas toujours au référentiel REN, mais nécessitent la prise en compte d'autres référentiels, structurés par la relation de rupture par rapport à REN. Prenons les exemples suivants

(a) Aujourd'hui, il fait meilleur qu'hier.

(b) Aujourd'hui, 2 janvier 2010, à 18 heures trente, se déroule...

(c) Ce jour-là, il faisait chaud, Luc marchait lorsqu'un orage éclata.

Dans (6a), le repérage est uniquement énonciatif ; l'intervalle temporel déterminé par hier prend sa valeur par rapport à aujourd'hui qui, lui, détermine un espace temporel englobant le processus de son énonciation. Dans (6b), l'ancrage référentiel se fait sur une date d'un référentiel (calendaire) externe à l'énonciation. Dans (6c), Ce jour-là est un marqueur linguistique qui est la trace d'une relation de rupture entre le référentiel énonciatif REN, où se situe l'énonciateur, et un autre référentiel, non actualisé par rapport à l'énonciation et dans lequel les situations verbalisées vont pouvoir être déterminées les unes par rapport aux autres.

\section{Exemples de référentiels non actualisés : RNA}

Bien qu'ils entretiennent certains rapports, les référentiels, que nous allons décrire sommairement, sont différents de la célèbre opposition de Benveniste (1966) entre «discours» et «histoire» ou de celle proposée par Weinreich (1973) entre « énonciation » et « narration » (voir aussi Fleishman, 1990). Ils ne doivent pas non plus être assimilés à «l'aoristique» de Culioli $(1980,1999)$. Dans de nombreux textes, on rencontre certaines relations prédicatives qui ne peuvent pas être repérées, par simple identification ou différenciation, par rapport à l'énonciation, c'est-à-dire par rapport au repère fixe ' $\mathrm{T}^{0}$ ' (dans REN).

Beaucoup de narrations ne sont situées ni dans le passé, ni dans le présent, ni dans le futur. Certains marqueurs explicites comme Ce jour là,... / Un jour,.../ Il était une fois... / Imagine la scène suivante... indiquent justement un non-repérage interne à REN et annoncent la création d'un autre référentiel où vont s'insérer les situations verbalisées. Il ne s'agit donc pas de «passés lointains » : un conte ou un mythe n'est pas situé dans le passé de l'énonciateur; d'autres marqueurs expriment ce décalage temporel (il y a plus de mille ans, il y a très longtemps, dans la préhistoire...). Certains procédés stylistiques peuvent certes entretenir une certaine confusion ${ }^{14}$ mais il nous paraît important de distinguer les différentes zones temporelles passées repérées par rapport à l'énonciation et donc faisant partie du référentiel REN, des zones temporelles de situations verbalisées qui ne peuvent plus être insérées dans le "réalisé » de l'énonciateur, ni situées dans un «à-venir » (Desclés \& Guentchéva, 2006). Ces situations font donc partie d'un autre référentiel que le co-énonciateur reconnaît immédiatement comme marqueurs linguistiques de changement de référentiels en rupture avec le référentiel énonciatif REN. Dans ce nouveau référentiel, qualifié de référentiel non actualisé et désigné par RNA, tous les instants différenciés les uns des autres sont en relation de rupture avec tous les instants de REN, donc en particulier avec ' $\mathrm{T}$ '. Les situations verbalisées et insérées dans RNA doivent donc être repérées les unes par rapport aux autres et non par rapport à $\mathrm{T}^{0}$ (Desclés 1984). Prenons un exemple où les situations évoquées relèvent manifestement d'un référentiel non actualisé RNA.

(7) Dans la plaine rase, sous la nuit sans étoiles, d'une obscurité et d'une épaisseur d'encre, un homme suivait seul la grande route de Marchiennes à Montsou; dix kilomètres de pavés coupant tout droit, à travers les champs de betteraves. Devant lui, il ne voyait même pas le sol noir, et il n'avait la sensation de l'immense horizon plat (...). L'homme était parti de Marchiennes vers deux heures. Il marchait d'un pas allongé, grelottant (...). Une seule idée occupait sa tête vide 
d'ouvrier sans travail et sans gîte, l'espoir que le froid serait moins vif après le lever du jour. Depuis une heure, il avançait ainsi, lorsque sur la gauche, à deux kilomètres de Montsou, il aperçut des feux rouges, trois brasiers brûlant au plein air, et comme suspendus. D'abord, il hésita, pris de crainte; puis, il ne put résister au besoin douloureux de se chauffer un instant les mains.

(Zola, Germinal)

Dans ce texte, les occurrences de l'imparfait et du plus-que-parfait renvoient à des valeurs aspectuelles d'état (n'avait pas, était parti) et de processus (suivait, ne voyait même pas, occupait sa tête, avançait) qui fixent ainsi un cadre temporel de référence narrative ; les événements qui se succèdent (aperçut, hésita, ne put résister) font irruption à l'intérieur de ce cadre en créant une structure narrative de succession : un événement succède à un autre événement dans le cadre déjà établi ; parfois un événement vient complètement modifier ce cadre. Aucun déictique ne vient donner une indication temporelle qui permettrait d'établir une relation quelconque avec le processus énonciatif qui pourtant prend en charge la narration.

Il est clair que certaines narrations viennent s'insérer dans le référentiel REN (Hier, écoute, voilà de qui m'est arrivé. Un homme apparemment ivre...). Par conséquent, la narration n'est pas spécifique au référentiel RNA, mais un grand nombre de narrations exprimées dans des romans, dans des contes, dans des fictions sont complètement détachées du référentiel REN et doivent être analysées comme telles.

\section{Synchronisation entre référentiels}

Si deux référentiels entretiennent des relations de rupture, ils peuvent néanmoins être synchronisés entre eux. C'est l'une des particularités du système général du repérage entre référentiels que de pouvoir justement synchroniser un instant d'un référentiel temporel avec un autre instant d'un autre référentiel temporel : deux instants t et $\mathrm{u}$ appartenant respectivement à deux référentiels en relation de rupture, c'està-dire [u \# t], peuvent être parallèlement synchronisés et donc identifiés, par la relation d'identification (l'identification ne signifie par identité !) : [u=t]. Il est donc possible de synchroniser un référentiel non actualisé RNA, dans lequel les situations d'une narration sont repérées les unes par rapport aux autres, avec le référentiel énonciatif REN qui les a pris en charge. Si de nombreuses narrations ne peuvent pas être situées par rapport à l'énonciation qui les raconte ${ }^{15}$, certaines indications permettent, parfois, de synchroniser la narration avec son énonciation comme, par exemple : L'histoire que je viens de vous raconter est ma propre histoire; ces événements, je les ai vécus moi-même, les personnages que j'ai évoqués, je les ai personnellement connus...

Il est également possible de synchroniser l'énonciation avec un référentiel externe, en identifiant la borne ' $\mathrm{T}^{0}$ ' avec un repère précis d'un système calendaire externe (aujourd'hui 25 juillet 2009...). Dans le référentiel RNA, les situations sont repérées les unes par rapport aux autres mais il est néanmoins possible de synchroniser les situations de la narration avec des zones temporelles d'un référentiel externe (par exemple, avec un calendrier), sans que, par ailleurs, ces situations soient explicitement repérables par rapport à l'énonciation qui les raconte (cette histoire s'est déroulée pendant le mois de juillet 1944...).

Le « présent historique », comme dans (7), fonctionne dans un référentiel narratif, souvent un référentiel RNA, dans lequel chaque événement est synchronisé, par l'énonciateur, avec le processus énonciatif.

$$
\text { (...) il prend le métro, descend à la Gare de Lyon, se précipite sur le quai } n^{\circ} 3 \text { : le train partait... }
$$

Plus précisément, les événements de la narration sont organisés d'un côté selon une structure narrative de succession mais, d'un autre côté, un instant constitutif, disons ' $t_{0}$ ', de la zone événementielle de l'événement raconté est synchronisé avec le repère ' $\mathrm{T}^{0}$ ' de REN : l'événement raconté est ainsi verbalisé sous la forme d'un processus inaccompli à l'instant ' $\mathrm{t}_{0}$ ', synchronisé avec ' $\mathrm{T}^{0}$ '; il est donc présenté comme étant en train de se dérouler « sous les yeux » de l'énonciateur-narrateur et de son co-énonciateurauditeur-lecteur (figure 3), d'où la vivacité stylistique du récit. 


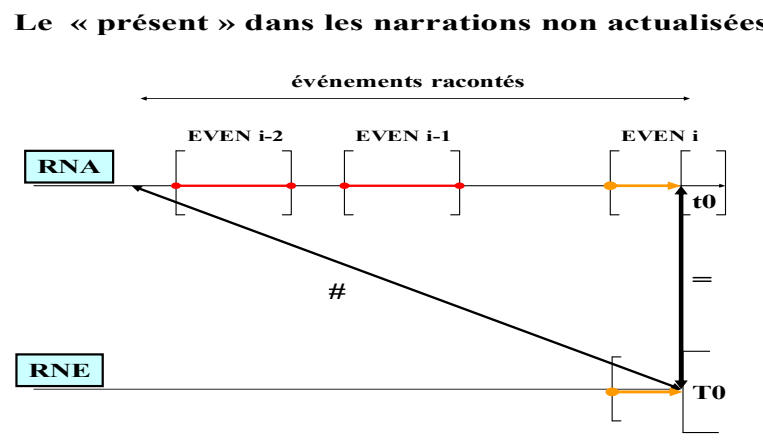

Figure 3 : Un événement raconté dans le référentiel RNA est présenté

comme se déroulant dans le référentiel REN à la borne d'inaccomplissement ' $\mathrm{T}_{0}$ '.

Le "présent de reportage », comme dans (9), introduit un autre type de synchronisation entre le référentiel énonciatif REN et le référentiel externe REX où se succèdent et se réalisent les événements énoncés en même temps que les processus qui les construisent (figure 4) :

(...) Zidane s'empare du ballon, ajuste son tir, tire et... rate le but: la France n'est plus championne du monde!

Chaque événement qui va avoir une occurrence dans le référentiel externe REX est aussitôt énoncé comme un processus en train de se dérouler dans le référentiel REN, sous la forme d'un processus inaccompli en ' $\mathrm{T}$ '.

Dans le présent historique, l'énonciateur synchronise la narration des événements avec sa propre énonciation, la narration se déroulant (fictivement car résultant d'une synchronisation) devant le coénonciateur; dans le présent de reportage, c'est l'énonciateur qui se synchronise avec la succession des événements externes, et il décrit ce qui se passe en insérant son énonciation dans le référentiel externe. En reprenant les différentes valeurs du présent, on peut faire apparaître un invariant aspectuel : c'est un inaccompli avec la borne ouverte à la droite du procès et avec une identification à un repère temporel : dans le référentiel REN, elle est identifiée au repère $\mathrm{T}^{0}$, ou, étant située dans un autre référentiel, avec un instant particulier de la narration (à ce moment là, il prend ses jambes à son coup et détale ....) ou encore, identifiée par synchronisation avec la borne $\mathrm{T}^{0}$. Ce qui caractérise le présent grammatical du français, c'est donc l'aspect inaccompli (état ou processus) et une identification.

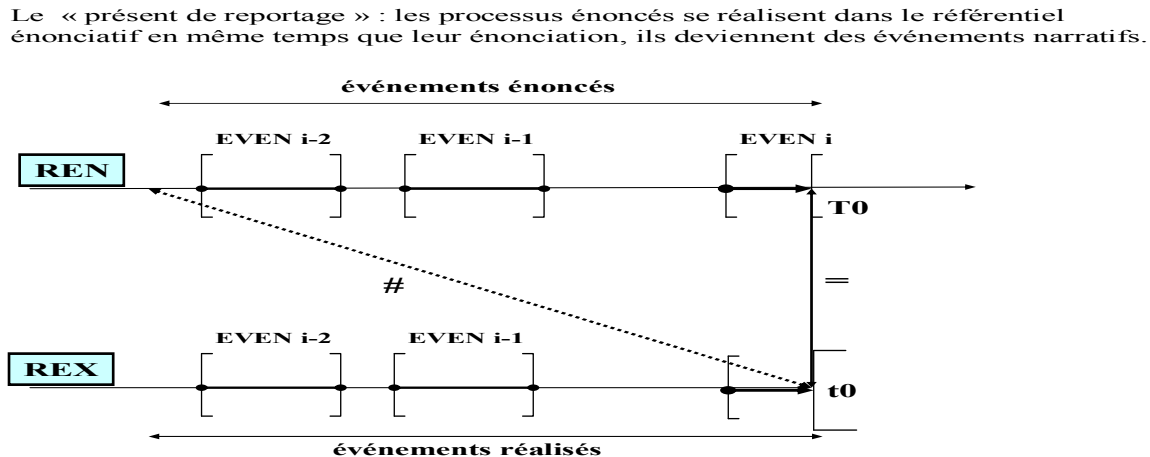


Figure 4 : Un événement qui a une occurrence dans le référentiel externe REX, est présenté, dans le référentiel énonciatif REN, sous la forme d'un processus inaccompli.

\section{Référentiels directement rapportés à REN2 et référentiels attribués à un tiers}

Prenons quelques exemples de " prises en charge énonciatives par un tiers »
(a)
Le procureur a affirmé : "X est coupable »
Le procureur a affirmé que Xétait coupable.
(c) Selon le procureur, $X$ serait (/ est) coupable.

Dans l'énonciation directement rapportée (10a), il y a création d'un autre référentiel, le référentiel REN2 des énonciations rapportées, dans lequel le procès « $\mathrm{X}$ est coupable » est pris en charge par un locuteur (le procureur) distinct de l'énonciateur. ${ }^{16}$ Dans le référentiel REN de l'énonciateur, il y a bien une prise en charge du rapport d'une assertion tenue par un locuteur mais cette assertion n'engage cependant pas l'énonciateur qui se contente de prendre en charge seulement les propos du procureur en les reproduisant fidèlement, ou du moins en les présentant comme tels. L'énonciation rapportée indirecte (10b) est différente puisque, ici, l'énonciateur ne reproduit plus fidèlement les dires du locuteur mais simplement un contenu, ce qui peut prêter à une certaine manipulation de la part de l'énonciateur et conduire ainsi à déformer les exacts propos tenus par le locuteur et entraîner souvent des polémiques (... mais je n'ai pas dit cela, du moins sous cette forme...). Par une énonciation indirecte, l'énonciateur se dégage du contenu rapporté mais il prend en charge en même temps, en partie du moins, la formulation retenue du contenu rapporté qu'il attribue au locuteur. Il s'agit donc, ici, de faire appel à un autre référentiel, le référentiel des propos attribués (par l'énonciateur) à un locuteur explicite (dans l'exemple le procureur). Il en est de même de l'énonciation (10c) qui implique le même genre de référentiel (un référentiel des croyances attribuées à un tiers); l'alternance seraitlest dans (10c) entraîne une plus ou moins grande adhésion de l'énonciateur par rapport au contenu attribué : avec serait, l'énonciateur ne dit rien sur son adhésion éventuelle aux propos du procureur; avec est, en revanche, l'énonciateur semble beaucoup plus en adéquation avec le jugement du procureur et ce jugement peut ensuite être repris plus facilement par l'énonciateur comme un fait avéré qui peut ainsi se projeter dans son propre référentiel REN. Ces énonciations rapportées directement ou attribuées à un tiers doivent être soigneusement distinguées d'une énonciation médiative comme (10d), Ainsi, X serait (donc) coupable, que nous allons analyser plus loin mais qui ne fait pas intervenir ce genre de référentiels (voir § 9).

\section{Référentiel des cadres de pensée RCP et référentiel des commentaires RCOM}

Dans un récit, le narrateur introduit souvent des pensées qui sont attribuées à l'un des actants de la narration, comme dans l'exemple suivant :

(11) Il s'arrête, il se met à rêver. Il se voit déjà en train de convaincre ses collaborateurs, de les entraîner dans son projet. Il reprend alors son chemin et, plein de confiance, il rejoint la salle de réunion.

Une analyse aspectuelle, effectuée à partir des occurrences des verbes conjugués, nous amène à annoter les propositions de ce texte à l'aide d'opérateurs aspectuels (d'événement ou de processus) ${ }^{17}$ : 
(10') $\quad$ EVEN $_{\mathrm{F} 1}\left(\mathrm{Il}\right.$ s'arrête), $\mathbf{P R O C}_{\mathrm{J} 2}\left(\right.$ il se met à $\left(\mathbf{P R O C}_{\mathrm{J} 3}(\right.$ rêver $\left.)\right)$. $\mathbf{P R O C}_{\mathrm{J} 4}(\mathrm{Il}$ se voit déjà en train de $\left(\mathbf{P R O C}_{\mathrm{J} 5}\right.$ convaincre ses collaborateurs $)$, de PROC $_{\mathrm{J} 6}($ les entraîner dans son projet $\left.)\right)$. $\mathbf{E V E N}_{\mathbf{F} 7}\left(\mathrm{Il}\right.$ reprend alors son chemin) et $\mathbf{E V E N}_{\mathbf{F 8}}$ (rejoint la salle de réunion $\left.{ }_{\mathrm{F}}\right)$.

On a l'énonciation d'un premier événement ' $\mathrm{EVEN}_{\mathrm{F} 1}$ (il s'arrête)', puis un processus ' $\mathrm{PROC}_{\mathrm{J} 2}$ (il se met à $\mathrm{PROC}_{\mathrm{J3}}($ rêver))' qui construit un autre référentiel, constituant ainsi un «cadre de pensée » $\mathbf{R C P}$ attribué au référent de $i l$. Le ' $\mathrm{PROC}_{\mathrm{J} 4}$ (il se voit)' décrit un processus de perception qui est réalisé dans le référentiel énonciatif $\mathbf{R E N}$ mais les processus ' $\mathrm{PROC}_{\mathrm{J5}}$ (en train de convaincre...)', ' $\mathrm{PROC}_{\mathrm{J} 6}$ (en train de les entrainer dans...)' ne se réalisent pas dans REN mais dans le référentiel RCP. Les événements ' $\mathrm{EVEN}_{7}$ (il reprend...)' et ' $\mathrm{EVEN}_{\mathrm{F} 8}$ (il rejoint...)' ont des occurrences situées à nouveau dans le référentiel REN (voir la figure 5) :

\footnotetext{
Il s'arrête un moment et se met à rêver. Il se voyait déjà en train de convaincre ses amis et de les entraîner dans son aventure. Il reprend, alerte, sa route et rejoint ses camarades qui l'attendaient.
}

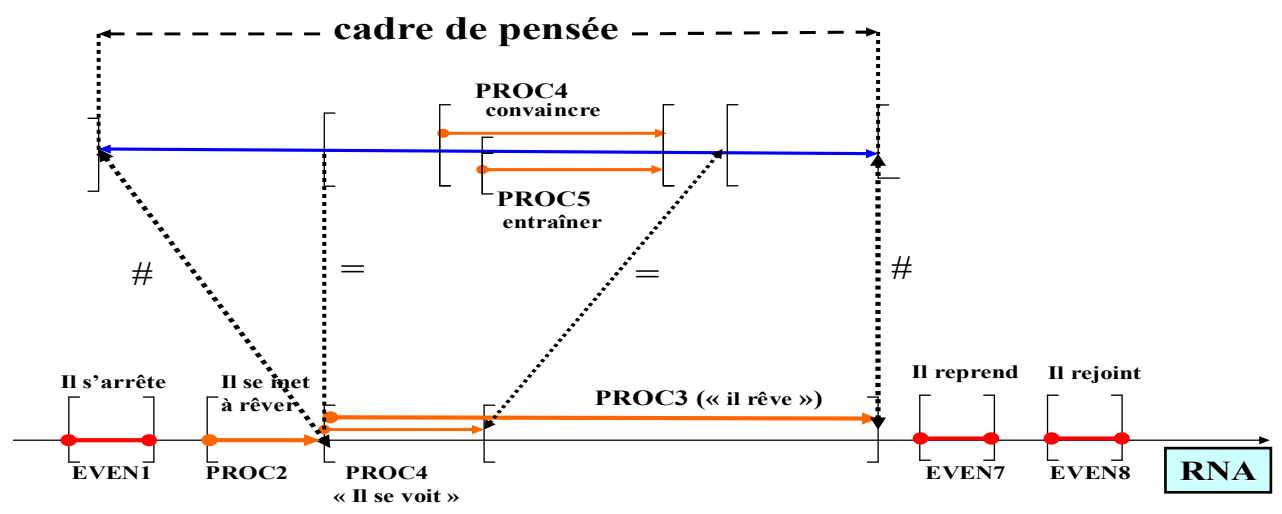

Figure 5 : Le « cadre de pensée » est construit par un processus (de représentation intellectuelle)

$$
\text { ' } \mathbf{P R O C}_{\mathbf{J} 2} \text { (il se met à } \mathbf{P R O C}_{\mathbf{J 3}} \text { (rêver))'. }
$$

Bien souvent le narrateur (que l'on peut distinguer de l'énonciateur) introduit des commentaires dans le texte qu'il produit. Ces commentaires peuvent être soit pris en charge par le narrateur, soit attribués à l'un des actants de la narration (c'est le problème bien connu du « style indirect libre »). Prenons, par exemple, le fragment du texte suivant :

Il partit. Le village était distant de $15 \mathrm{~km}$. Il lui fallait donc environ 3 heures pour le rejoindre. Il accéléra le pas.

avec l'annotation aspectuelle qui en résulte :

$\mathbf{E V E N}_{\mathbf{F 1}}$ (il partit). $\mathbf{E T A T}_{\mathbf{O} 2}$ (le village était distant ...). $\mathbf{P R O C}_{\mathbf{J 3}}$ (il lui fallait...) pour $\mathbf{E V E N}_{\mathbf{F} 4}$ (le rejoindre)). $\mathbf{E V E N}_{\mathrm{F} 5}$ (il accéléra...).

L'imparfait le village était distant d'environ... introduit ici un commentaire dont la prise en charge peut être soit celle de l'actant, auquel cas le référentiel créé serait un "cadre de pensée » (les pensées de l'actant du récit), soit celle du narrateur qui donne ainsi des indications utiles au lecteur pour une 
meilleure compréhension du texte. Dans les deux cas, les informations ne font pas partie du référentiel RNA où se succèdent les situations verbalisées dans la narration.

L'utilisation d'un proverbe ${ }^{18}$ comme :

\section{Bien mal acquis ne profite jamais}

exprime une actualisation d'une vérité générale tirée de l'expérience collective qui s'intègre dans le référentiel des vérités générales RVG : pour chaque instant de ce référentiel, cette vérité générale est considérée comme vraie ; une occurrence de cette relation prédicative étant projetée, par synchronisation, dans un autre référentiel, RNA ou REN selon les cas, elle est alors considérée comme une instance particulière qui est réalisée dans l'un de ces référentiel.

\section{Référentiels des situations possibles RPOS}

Prenons quelques exemples simples, introduits par le connecteur si, que nous allons chercher à différencier par des analyses et représentations différentes :
(a) Si tu fais un pas de plus, je tire!
(b) S'il a de l'argent, il te le donne / il te le donnera
(c) S'il avait de l'argent, il te le donnerait
(d) Si, à cette époque, il avait eu de l'argent, il te l'aurait donné
(e) Si, à cette époque, il avait (eu) de l'argent, il te le donnait

Le si hypothétique est une instruction de construction d'un autre référentiel, le référentiel RPOS des situations possibles. Dans ce référentiel, les situations et les relations entre situations sont seulement envisagées comme étant possibles et, de ce fait, elles ne sont pas nécessairement réalisées dans le référentiel REN (ou, dans une narration impliquant le référentiel RNA). Elles peuvent être cependant confrontées, par une synchronisation, au référentiel REN (ou aux situations du référentiel RNA) où se réalisent effectivement (et non potentiellement) les procès.

Dans (14a), il s'agit d'une menace actuelle relevant du référentiel REN ; la relation entre deux processus, $\operatorname{PROC}_{\mathrm{J} 1}$ (tu fais un pas de plus) et sa conséquence immédiate et concomitante $\operatorname{PROC}_{\mathrm{J} 2}$ (je tire), avec $\left[\mathrm{J}_{2} \subseteq\right.$ $\left.\mathrm{J}_{1}\right]$, est posée et insérée dans le référentiel RPOS; or, comme le premier processus n'est pas effectivement (encore) réalisé dans le référentiel REN (tu ne fais pas un pas de plus), il s'ensuit que le second (je tire) ne l'est pas (encore), d'où, cependant, la valeur de menace ${ }^{19}$.

Dans (14b), il y a une confrontation entre ce qui est posé dans le RPOS (concomitance d'un état présent - il a de l'argent - avec le processus - il te le donne) d'un côté et l'état qui, lui, est éventuellement réalisable dans le référentiel REN, d'un autre côté. Tant que l'on n'a pas vérifié que l'état est effectivement réalisé, le processus ne se réalise pas maintenant ( $s$ 'il a de l'argent maintenant, il te le donne) ou ne se réalisera pas, plus tard (s'il a de l'argent maintenant, il te le donnera plus tard $)^{20}$.

Avec (14c), il y a une confrontation contrefactuelle entre un état possible (dans le RPOS) et l'état effectivement constaté dans le REN ; il s'ensuit que le processus posé comme étant concomitant, dans le RPOS, à l'état contrefactuel, ne peut pas être réalisé dans le REN ${ }^{21}$, d'où le conditionnel et l'inférence qui s'en déduit (il ne te donne pas d'argent) (voir figure 6).

Avec (14d), il s'agit de la trace d'un raisonnement : la concomitance entre un état et un processus a été posée dans le RPOS ; en synchronisant le réalisé passé exprimé dans le REN avec un état possible situé dans le RPOS, on constate qu'il y a une contradiction par rapport à ce que l'on connait du passé : si l'état (il avait eu de l'argent) qui, consécutif à un certain événement passé (par exemple, un contrat d'achat a été signé), avait été réalisé, alors l'état résultant (il te l'aurait donné) se serait réalisé également ; or, on sait que, dans le réalisé passé, l'état (il avait de l'argent) n'a pas été réalisé ; il s'ensuit donc, par inférence, que l'événement (il t'a donné de l'argent) et son état résultant n'ont pas eu lieu ${ }^{22}$. 
L'énoncé (14d) donne l'information suivante: il était bien possible que l'on obtienne de sa part de l'argent mais, comme nous le savons bien tous les deux, à cette époque là, il n'avait pas d'argent et donc il n'a pas pu lui en donner.

Une autre valeur de (14d), de nature plus explicative, est cependant également envisageable et elle semble plus apparente avec l'énonciation de (14e) : une implication entre l'état et l'événement est posée dans le RPOS ; grâce à la connaissance du passé réalisé, on sait que l'événement n'a pas eu lieu (on sait tous les deux qu'il n'a pas eu de l'argent de sa part), et, par conséquent, il en est de même du processus qui engendre cet événement et de l'état qui en résulte. A la suite d'une synchronisation avec le passé dans le REN, une explication peut être inférée (il n'avait pas d'agent à cette époque) par simple contraposition ${ }^{23}$ (voir la figure 6).
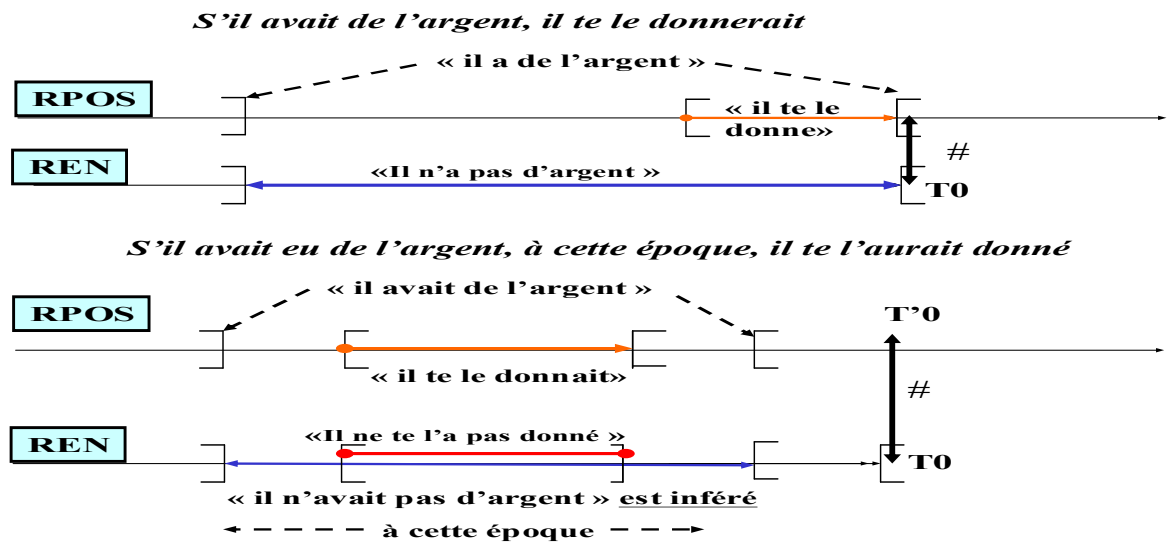

Figure 6. si, marqueur d'un référentiel RPOS

Dans l'analyse des exemples (14a) - (14d), il s'agit d'une équivalence posée dans le RPOS, alors que dans l'analyse de (14e), c'est une implication.

L'analyse sémantique de si est souvent fort complexe. Prenons deux exemples, l'un emprunté à O. Ducrot, l'autre à O.W. Quine : (a) Si tu as soif, il y a de la bière dans le réfrigérateur ; (b) Vous êtes reçu si vous avez payé vos droits d'inscription. Le premier énoncé (a) ne doit pas être analysé par l'implication 'si $\mathrm{p}$ alors q' avec $\mathrm{p}=$ «tu as soif» et $\mathrm{q}=$ «il y a de la bière dans le réfrigérateur » puisque la contraposée, qui doit lui être équivalente, signifie autre chose : «s'il n'y a pas de bière alors tu n'as pas soif !». L'analyse sémantique correcte de (a) est plutôt 'q si p', soit encore 'si q alors p' (il y a de la bière si tu as soif). L'énoncé (b) s'analyse par l'implication 'si $\mathrm{p}$ alors q', avec $\mathrm{p}=$ " vous êtes reçu » et $\mathrm{q}=$ « vous avez payé vos droits» et non pas par 'si q alors p'. Les implications correctes sont posées dans RPOS et lorsque l'antécédent est réalisé dans REN, le conséquent s'en déduit immédiatement dans REN.

Prenons maintenant l'énoncé suivant :

$$
\text { Si son père avait été absent, Marie partait. }
$$

A partir de l'énoncé (15), nous pouvons inférer :

\section{Son père n'était (donc) pas absent.}

L'énonciateur pose ainsi, dans le RPOS, une relation d'implication entre un état (son père est absent) et un processus (Marie part) qui en est une des conséquences immédiates. Or, avec (15), il est énoncé que, dans le référentiel énonciatif REN, l'événement passé (Marie est partie) n'a pas eu lieu. Ainsi, par contraposition et modus ponens, l'état n'a donc pas été réalisé dans le passé, d'où l'inférence ${ }^{24}$ qui résulte de la compréhension de (15) : son père était présent. 


\title{
10 Référentiel des connaissances partagées RCP
}

Considérons maintenant les deux exemples suivants :

\author{
(a) Il a donc plu [puisque la route est mouillée] \\ (b) Ainsi, il aurait plu \\ (c) Il aurait (donc) plu
}

Les énonciations en (16) s'appuient sur : $1^{\circ}$ ) un constat : « la route est mouillée »; $2^{\circ}$ ) un savoir commun partagé avec le co-énonciateur: "s'il pleut, alors la route est généralement mouillée»; $3^{\circ}$ ) un raisonnement abductif qui remonte, à partir de l'état constaté, vers une cause plausible (l'événement « il a plu »); $4^{\circ}$ ) l'affirmation de la concomitance entre l'état constaté (« la route est mouillée ») et l'état résultant de l'événement considéré comme plausible; $5^{\circ}$ ) l'énonciation de l'événement reconstruit et présenté comme étant seulement plausible au moyen de traces linguistiques qui, en français, sont par exemple donc, ainsi, ou encore l'utilisation de la forme conditionnelle (Figure 6). Cette énonciation s'oppose alors à l'énonciation il a plu qui renvoie à la simple occurrence d'un événement ou de son état résultant; elle s'oppose également à l'énonciation du simple constat. Les énonciations en (16) sont en fait le résultat de raisonnements abductifs qui se signalent comme tels au co-énonciateur. Si l'implication entre un événement et l'état qui en est une conséquence est posée dans le référentiel des savoirs partagés RSP, la prise en charge, à partir d'indices constatés, d'un événement jugé seulement plausible justifie un certain désengagement de l'énonciateur dans le REN. Ce désengagement relève de ce qu'on peut appeler «la catégorie du médiatif», appelée parfois «évidentialité ». Devant ce genre d'énoncés, le coénonciateur peut en effet répliquer: Mais non, il n'a pas plu; si la route est mouillée (compréhension du savoir partagé évoqué), c'est parce que l'arroseuse municipale est passée dans cette rue il y a quelques minutes. Ainsi, les énonciations comme celles de (15) ne ferment-elles pas une éventuelle négociation dialogale, comme le ferait, en revanche, une assertion comme : Je te l'affirme : il a plu!

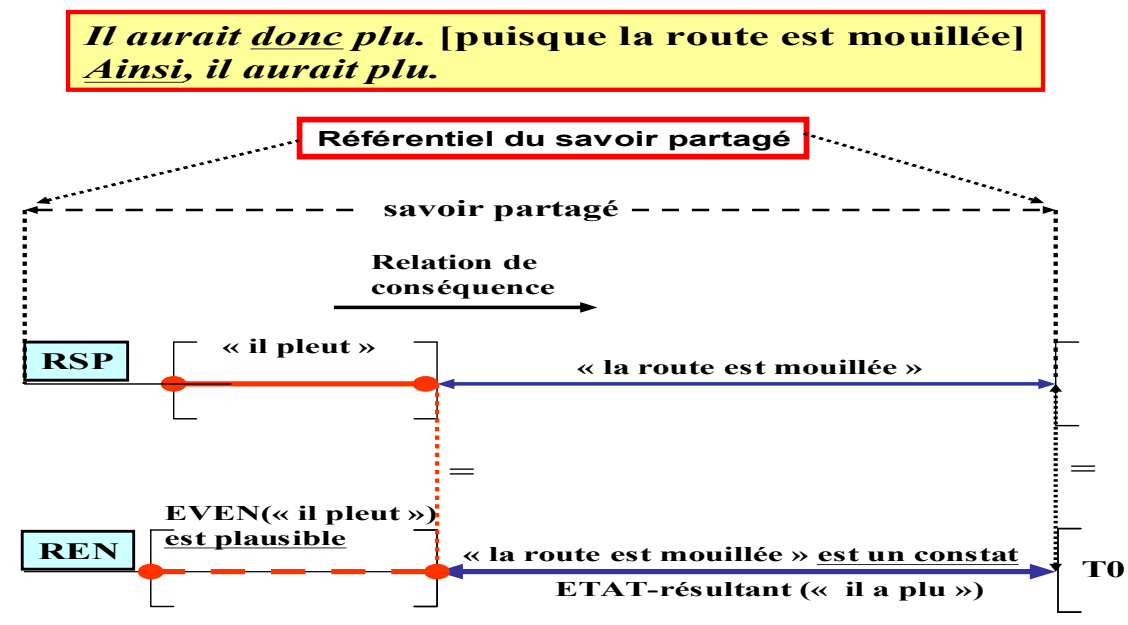

Figure 7 : Confrontation entre un constat d'observation dans le REN avec un savoir partagé commun ; cela conduit à l'énonciation d'un état résultant d'un événement présenté comme simplement plausible.

Remarque : Le raisonnement abductif a été introduit par C. S. Peirce (et, indépendamment par G. Polya (1989), sous le nom de "syllogisme hypothétique »). Peirce (1974) distingue le raisonnement déductif (par exemple, par le schème de modus ponens) du raisonnement abductif dont la forme canonique est: $1^{\circ}$ ) $\mathrm{H}$ (hypothèse) $=>\mathrm{C}$ (conclusion), extraite d'un savoir partagé entre l'énonciateur et son co-énonciateur (par exemple, une vérité générale); $2^{\circ}$ ) or, $\mathrm{C}$ a été constaté ; $3^{\circ}$ ) donc, $\mathrm{H}$ est jugée plausible (l'hypothèse $\mathrm{H}$, plausible, devient ainsi une éventuelle explication du constat $\mathrm{C}$ ). 


\section{Nécessité d'introduire différents types de référentiels}

Nous avons montré que la conceptualisation de la temporalité opérée par les langues (dans nos exemples, le français) ne peut pas prendre pour modèle interprétatif sous-jacent un axe linéaire où le « présent»" viendrait s'insérer entre « le passé » et « le futur ». Notre analyse s'accorde bien avec la position adoptée par W. Klein (2009). Il apparaît que le temps grammatical du présent, par exemple, ne peut pas être toujours défini par rapport au processus énonciatif, il suffit de se reporter à nos analyses du présent narratif ou historique et à celle du présent de reportage. De même, l'imparfait n'est pas toujours un temps qui renvoie à une situation passée (qu'est-ce qu'on faisait déjà demain ?). Quant au temps grammatical du passé simple, il n'exprime pas toujours du passé puisque ses emplois situent plutôt des événements dans le référentiel RNA (par exemple, Lyons 1970 ; Comrie 1976 ; Mourelatos 1978)dont les événements ne sont pas reliés à l'énonciation. L'analyse de quelques emplois de si a bien montré comment fonctionnaient à la fois la relation de rupture (création d'autres référentiels) et celle de synchronisme entre le référentiel des situations possibles RPOS et le référentiel énonciatif REN : comment expliquer les inférences interprétatives qui en découlent, sans recourir à de telles relations et aux différents référentiels ? Si on analysait des textes on verrait très vite l'intrication des référentiels (commentaires du narrateur, situations évoquées comme possibles mais non-réalisées dans la narration, situations contrefactuelles, attribution de propos et croyances à un tiers sans que l'énonciateur s'engage à leur propos, appel à des vérités générales...). La dimension du présent article ne le permet pas car, avant d'analyser un texte, il faut présenter les outils conceptuels de son analyse, et tel était notre objectif.

Les invariants des temps grammaticaux présent / imparfait / passé composé / passé simple de l'indicatif ${ }^{25}$ en français s'opposent entre eux et sont décrits en faisant intervenir des différences de référentiels. Précisons, selon une formulation encore approximative, leurs invariants sémantiques :

- Le présent et l'imparfait sont des formes aspectuelles d'inaccompli (état ou événement) réalisées sur des intervalles temporels dont les bornes droites sont toujours ouvertes.

- La forme du présent est associée à une opération d'identification avec la borne $\mathrm{T}^{0}$, ou à une identification par synchronisme entre référentiels (présent historique présent de reportage, vérités générales ...).

- La forme de l'imparfait est associée à une opération de non-identification soit par différenciation (antériorité le plus souvent) dans un référentiel (REN ou RNA), soit par un marquage explicite d'un changement de référentiels (par exemple : recours à une énonciation rapportée implicite, soit encore à un commentaire ou encore à une situation contrefactuelle...). En dépit des apparences, un imparfait ne renvoie pas à un événement ${ }^{26}$ mais essentiellement à des états ou à des processus.

- Le passé simple est une forme qui, dénotant toujours un événement, relève du référentiel non actualisé RNA et, pratiquement jamais, en français contemporain, du référentiel énonciatif REN ; son emploi peu fréquent dans l'oral s'explique puisque l'oralité est étroitement liée à des dialogues relevant du référentiel énonciatif REN ou de référentiels de propos rapportés REN2.

- Le passé composé est une forme aspectuelle d'accompli, ce qui exclut la valeur de processus (inaccompli); cette forme renvoie dans le référentiel énonciatif REN soit à un événement déjà réalisé (donc accompli), soit à un état résultant (d'un événement déjà accompli) qui se réalise dans le présent de l'énonciation; cette valeur d'accompli-présent est associée aux valeurs aspectuelles traditionnelles du « parfait» dans certaines langues. Dans le référentiel REN, le passé composé permet de passer d'une valeur résultative à la valeur événementielle qui la précède immédiatement, d'où sa tendance, pour exprimer un événement, à remplacer la forme aoristique du passé simple. Ces deux valeurs, ainsi que les valeurs dérivées comme le parfait d'expérience (exemples : il a vécu à Londres, il connaît donc bien cette ville / il a fait une thèse, il sait donc ce que cela implique pour un jeune chercheur ...) dans le REN comme dans d'autres référentiels, en particulier dans le RNA, dépendent étroitement du contexte (l'événement et l'état résultant sont alors repérés par rapport à un instant pris pour référence dans une narration). 
En introduisant différents types de référentiels dans l'analyse des marqueurs aspecto-temporels, nous sommes conduits à des analyses cohérentes, et sur le plan cognitif, relativement simples et naturelles, ce qui permet de rattacher l'analyse du temps appréhendé au travers de ses catégorisations par les langues, aux analyses entreprises par la physique (temps de l'univers), la biologie (temps de la vie), la psychologie (temps de la conscience). Cette étude sur les référentiels s'inscrit comme une des pièces majeures d'une théorie plus générale des représentations aspecto-temporelles en français et dans d'autres langues (bulgare, russe, polonais, coréen, arabe...).

\section{Références bibliographiques}

Alrahabi, M. (2010). Excom-2 : plateforme d'annotation automatique de catégories sémantiques: Conception, modélisation et réalisation informatique. Applications à la catégorisation des citations en arabe et en français. Thèse de doctorat, Université Paris-Sorbonne, 2010.

Anscombre, J.C. (2006). Polyphonie et classification des énoncés sentencieux, Le Français Moderne, 1, 87-99.

Benveniste, E. (1966). Problèmes de linguistique générale, Vol. I, Paris: Gallimard.

Benveniste, E. (1974). Problèmes de linguistique générale, Vol. II, Paris: Gallimard.

Berthonneau, A.-M. et Kleiber G. (1994). Imparfaits de politesse : rupture et cohésion, Travaux de linguistique, 29, 59-92.

Berthonneau, A.-M. et Kleiber G. (1999). Pour une réanalyse de l'imparfait de rupture dans le cadre de l'hypothèse anaphorique méronimique, in Brès J. et al., L'imparfait dit narratif, langue, discours, Cahiers de praximatique, $10-166$.

Chevalier, J.-C., Blanche-Benveniste, C., Arrivé, M. et Pétard, J. (1964). Grammaire Larousse du français contemporain, Paris : Larousse.

Comrie, B. (1976. Aspect. An introduction to the study of verbal aspect and related problems. Cambridge : Cambridge University Press.

Comrie, B. (1985). Tense. Cambridge: Cambridge University Press.

Culioli, A. (1980). Valeur aspectuelle et opérations énonciatives: l'aoristique, in J. David et R. Martin (eds) : Notion d'aspect, Paris: Klincksieck, 181-193.

Culioli, A. (1999). Pour une linguistique de l'énonciation, Formalisation et opérations de repérage, t. 2, Paris : Ophrys.

Desclés, J.-P. (1980). Construction formelle de la catégorie de l'aspect (essai), in J. David et R. Martin (éds), $L a$ notion d'aspect, Paris: Klincsieck, 195-237.

Desclés, J.-P. (1984). Quelques concepts relatifs au temps et à l'aspect pour l'analyse des textes, Studia Kognitywne, I, Varsovie : Académie des sciences de Pologne, 57-88.

Desclés, J.-P. (1989). State, Event, Process and Topology, General Linguistics 3/29, The Pennsylvania University Press, University Park and London, 159-200.

Desclés, J.-P. (1995). Les référentiels temporels pour le temps linguistique, Modèles linguistiques, XVI, 2, 9-36.

Desclés, J.-P. (2003). Imparfait narratif et imparfait de nouvel état en français, Etudes linguistiques romano-slaves offertes à Stanislaw Karolak, Cracovie: Wydawnicza "Edukacija", 131-155.

Desclés, J.-P. (2004). Reasoning and aspecto-temporal calculus, in D. Vanderveken (éd.): Logic, Thought and Action, Dordrecht: Kluwer Publisher, 217-244.

Desclés, J.-P. et Z. Guentchéva (1995). Is the Notion of Process Necessary?, in Bertinetto P.M. and alii (eds), Temporal Reference, Aspect and Actionality, Turin : Rosenberg \& Sellier, 55-70.

Desclés, J.-P. \& Z. Guentchéva. Convergences et divergences dans quelques modèles du temps et de l'aspect, Semantyka a konfrontacija jezykowa, 1, Varsovie (Pologne), 23-41. 
Desclés, J.-P. et Z. Guentchéva. (2006), Référentiels aspecto-temporels dans les textes, Studia Kognitywne, 7 , Varsovie: Académie des sciences de Pologne \& Instytut Slawistyki, 11-38.

Desclés, J.-P. et Z. Guentchéva. (2008). Analyse sémantique des Temps, Aspects, Modalités (TAM) par des représentation topologiques. The 18th International Congress of Linguistics (abstracts), July 21-26, 2008, Korea University, Seoul, Korea.

Gosselin, L. (1996). Sémantique de la temporalité en français (Un modèle calculatoire et cognitif du temps et de l'aspect), Duculot.

Fleischman, S. (1990). Tense and Narrativity (From Medieval Performance to Modern Fiction), Austin: University of Texas Press.

Guentchéva, Z. (1990). Temps et aspect: l'exemple du bulgare littéraire contemporain, Collection Sciences du Langage, Paris: Presses du CNRS.

Karolak, S. (1997). Le temps et le modèle de H. Reichenbach, in Etudes cognitives, 2, Varsovie: Académie des sciences de Pologne \& Instytut Slawistyki, 95-125.

Kleiber, G. (1999). Les proverbes : des dénominations d'un type très très spécial, Langue française, 123, 52-69.

Klein, W. (2009). How time is encoded, in W. Klein \& P. Li, The expression of time, Berlin: Moutons de Gruyter, 3982.

Lyons, J. (1970). Linguistique générale. Introduction à la linguistique théorique, Larousse.

Martin, R. (1981). Le futur linguistique : temps linguistique ou temps ramifié (A propos du futur et du conditionnel français), Langages, 64, 81-92.

Mourelatos, A. (1978). Events, processes and states, Linguistics and Philosophy, 2, 415-434.

Weinreich, H. (1973). Le temps, Le récit et le commentaire, trad. de l'allemand par M. Lacoste (original 1964). Paris: Seuil.

Pierce, C. (1974), Collected Papers, Vol. I-VI. Cambridge, Massachusetts: The Belknap Press of Harvard University Press.

Polya, G. (1989). Comment poser et raisonner. Un problème, Editions Jacques Gabey.

Provôt, A., Desclés J.-P. \& Vinzerich A. (sous presse). Invariant sémantique du présent de l'indicatif en français, Cahiers Chronos 2010, Amsterdam \& New York: Rodopi.

Reichenbach, H. (1947). Elements of Symbolic Logic, London: Collier-Macmillan ; Reprinted in J.M. Moravcsik (editor), 1974, Logic and Philosophy for Linguists, A Book of Readings, The Hague : Mouton, 122-141.

Saint Augustin (1993). Confessions, Edition de Philippe Sellier, Paris: Gallimard.

Touratier, Ch. (1996). Le système verbal français (Description morphologique et morphématique), Paris : Armand Colin.

Vuillaume, M. (2001). L'expression du futur dans le passé en français et en allemand, in P. Dendale et L. Tasmowski, Le conditionnel en français, Metz : Université. de Metz (diffuseur: Klincksieck), 105-123.

Wagner, R.L. et Pinchon, J. (1962). Grammaire du français classique et moderne, Paris : Hachette.

Weinrich, H. (1973). Le Temps, Paris : Le Seuil.

Wilmet, M. (1997). Grammaire critique du français, Duculot.

\footnotetext{
${ }^{1}$ L'aspectualité n'est donc pas réduite à l'aspect verbal, comme semble le faire apparaître la morphologie de nombreuses langues. L'aspect est analysé comme un opérateur dont une relation prédicative, prise dans sa globalité, est l'opérande, constituant ainsi un procés; ce dernier doit être relié aux conditions mêmes de son énonciation. L'analyse détaillée de l'aspectualité, que nous ne faisons pas ici, fait intervenir également une aspectualité relative au prédicat verbal (l'aspect lexical), puisque le prédicat verbal contient du temps, et également une relation entre le prédicat verbal et un des actants de la relation prédicative, selon que cet actant est complètement ou partiellement
} 
affecté (il a peint sa chambre en une heure / il a peint une chambre pendant une heure) ou entièrement atteint ou non (il est monté sur la colline / il monte la colline)...

${ }^{2}$ Ces trois distinctions aspectuelles de base sont constitutives de l'opposition, importante mais trop générale, entre «accompli» et «inaccompli», que l'on ne doit pas confondre avec l'opposition "perfectif» (avec la valeur sémantique d'achèvement) et «imperfectif», opératoire pour la description des langues slaves. Aux trois distinctions de base, il faut ajouter la notion aspectuelle de suite discrète d'occurrences de situations identiques, classe qui peut être ouverte (sans dernière situation) ou fermée (avec une dernière situation). Pour désigner une situation référentielle à laquelle renvoie un énoncé, nous préférons le terme de « situation » (verbalisée) à celui d' «état de chose », puisque toute situation n'est pas toujours un état, ou à celui trop générique d' événement » (référentiel) puisque toute situation n'est pas nécessairement un événement.

${ }^{3}$ Les recherches épistémologiques sur le temps et les notions de mouvement et de changement ont été entreprises par Aristote (Physique), puis reprises par Galilée avec la formulation des premiers principes de la relativité, et poursuivies par H. Poincaré et A. Einstein avec la relativité restreinte.

${ }^{4}$ Pour une critique du modèle de H. Reichenbach, voir notre article (Desclés \& Guentchéva, 1996).

${ }^{5}$ Mentionnons par exemple S. Karolak (1997).

${ }^{6}$ Techniquement, il s'agit d'une coupure continue au sens de Dedekind. Un élément est une coupure continue entre une partition de deux ensembles ordonnés $A_{1}$ et $A_{2}$ (par exemple les instants réalisés et les instants non réalisés), qui se succèdent (tout instant de $A_{1}$ est antérieur à tout instant de $A_{2}$ ), lorsque : (i) il n'y a ni saut - donc absence de 'trou temporel' entre $\mathrm{A}_{1}$ et $\mathrm{A}_{2}$; (ii) ni lacune - donc absence d'un quelconque instant lacunaire qui viendrait s'intercaler entre $A_{1}$ et $A_{2}$ (c'est-à-dire entre le réalisé et le non réalisé) ; et (iii) lorsque la frontière, qui constitue la coupure, entre $A_{1}$ et $A_{2}$ est soit l'élément le plus grand de $A_{1}$ (ou le dernier élément de $A_{1}$ ) mais alors pas le plus petit de $A_{2}$, soit la borne n'est pas le plus grand élément de $A_{1}$ mais le plus petit de $A_{2}$ (ou le premier élément de $A_{2}$ ). Dans le cas de la borne $\mathrm{T}^{0}$, il s'agit bien d'une borne frontière qui est une coupure continue, à condition de supposer que la temporalité est continue (les instants peuvent être mesurés par des nombres réels) et non discrète (dans ce cas, les instants ne peuvent être énumérés que par des nombres entiers) : $T^{0}$ vient couper la temporalité sous la forme d'une borne d'inaccomplissement dans le réalisé ; elle n'est donc pas un instant réalisé mais le premier instant du non réalisé (voir Desclés 1978, 2005). Il s'ensuit que deux événements qui se suivent ne peuvent jamais être contigus, que la zone temporelle contiguë à un événement est toujours un ouvert (zone de réalisation d'un état).

${ }^{7}$ La notion de temps ramifié apparaît chez R. Martin (1981) et M. Vuillaume (2001), entre autres. Cependant, chez ces auteurs, l'instant T0 n'est pas considéré comme une borne inaccomplie de l'énonciation, c'est-à-dire le premier instant du non-réalisé, ce qui nous permet de relier l'aspectualité à la modalité.

${ }^{8}$ Voir à ce propos Chevalier et al. $(1964: 338, \S 477):$ « [...] le présent y traduit "aidé par le contexte [...] un passé récent" comme dans : Il quitte à l'instant son ami », ainsi que L. Wagner et J. Pinchon (1962 : 345, § 399) pour qui le présent admet «d'être déterminé par des compléments qui évoquent le début ou la fin de cette durée ».

\footnotetext{
${ }^{9}$ Saint Augustin, pour qui « le présent» ne pouvait pas «être long», ne situait pas son analyse dans le référentiel énonciatif REN mais dans le référentiel externe REX, dans lequel la projection de $\mathrm{T}^{0}$ est un instant $\mathrm{t}_{\mathrm{m}}$ mobile.

${ }^{10}$ Dans l'analyse de l'état résultant, la borne de la fin de la transition événementielle est une coupure continue entre l'intervalle fermé de transition de l'événement et l'intervalle ouvert qui lui est contigu et postérieur; cette borne appartient par conséquent au premier intervalle mais pas au second. Il est donc difficile de rendre compte de l'état résultant sans faire intervenir la notion de coupure continue. Par exemple, le système de Reichenbach ne rend pas compte adéquatement de cette valeur aspectuelle et de l'opposition fondamentale entre l'événement et l'état résultant contigu et immédiatement postérieur, excluant ainsi tout saut et tout élément lacunaire.

${ }^{11}$ Nos analyses et, bien sûr, les représentations qui en découlent, sont assez différentes de celles de L. Gosselin (1996) qui n'utilise pas la topologie des intervalles et donc les notions de bornes ouverte ou fermée, essentielles selon nous pour rendre compte des oppositions aspectuelles, en particulier, entre inaccomplies et accomplies. De plus, la notion de processus est absente dans son approche théorique.

${ }^{12}$ Berthonneau \& Kleiber (1994) expliquent cet emploi de l'imparfait de la façon suivante : « [il] renvoie à une situation du passé, à savoir celle où la cliente attendait que le commerçant puisse la servir » (p. 74) et « le locuteur signale en somme à l'interlocuteur que celui-ci était mentalement présent avant même qu'on s'adresse à lui » (p. 76).
} 


\footnotetext{
${ }^{13}$ Nous allons spécifier plus loin l'invariant de l'imparfait. Quant à l'invariant du présent de l'indicatif, nous renvoyons à Provôt et al. (sous presse).

${ }^{14}$ Rappelons à ce propos les études de M. Vuillaume (1990, 1993) concernant l'emploi des déictiques temporels comme aujourd'hui ou maintenant dans le roman du $\mathrm{XIX}^{\mathrm{e}} \mathrm{s}$. où un passé simple peut se trouver en cooccurrence avec le déictique aujourd'hui. Selon l'analyse de l'auteur, si le passé simple renvoie à un événement qui, coupé de l'instance d'énonciation, est repéré par rapport aux événements racontés, aujourd'hui établit un autre repérage que celui défini par le passé simple : il est associé au couple narrateur / lecteur et renvoie au moment de la lecture de l'histoire. En ce qui nous concerne, aujourd'hui, dans le référentiel RNA, désigne un index temporel de lecture qui est indépendant de l'énonciateur et on peut considérer que, dans ce contexte, il n'a pas la valeur de déictique centré sur l'acte d'énonciation.

${ }^{15}$ Il faut bien entendu distinguer « le narrateur » qui laisse des traces explicites dans la narration et l'énonciateur (l'auteur de la narration); dans certains cas, le narrateur et l'énonciateur se confondent, dans d'autres cas, ils sont dissociés.

${ }^{16}$ La distinction entre énonciateur et locuteur est fondamentale pour l'étude des discours rapportés (Desclés, 1980). Cependant, elle ne doit pas être assimilée à la distinction proposée par O. Ducrot (voir sur ce point: Desclés \& Guentchéva, 2000 et Desclés, 2009). Pour un traitement automatique des discours rapportés avec toutes leurs différentes valeurs, voir la thèse de M. Alrahabi (2010).

17 Par la technique d'exploration contextuelle, on peut mettre en jeu une procédure automatique d'annotation des valeurs aspectuelles des propositions utilisant la plate-forme EXCOM 2, développée dans le cadre de l'équipe LALIC de l'Université Paris-Sorbonne.
}

\footnotetext{
${ }^{18}$ Les approches linguistiques sur les proverbes se sont multipliées au cours de ces trois dernières décennies. On oppose généralement la thèse dénominative (Kleiber 1999) et la thèse polyphonique (Anscombre 2006). L'utilisation de la notion de référentiel et de sa relation avec un autre référentiel donne, nous semble-t-il, une explication plus économique et plus unifiée.

${ }^{19}$ On peut formaliser ce raisonnement comme suit : $1^{\circ}$ ) dans RPOS nous avons l'équivalence suivante : 'PROC $\mathrm{J}_{1}(\mathrm{p})$ $\Leftrightarrow \operatorname{PROC}_{\mathrm{J} 2}(\mathrm{q})$ ' avec $\left[\mathrm{J}_{2} \subseteq \mathrm{J}_{1}\right]$; or, à la suite de la synchronisation $\left[\mathrm{J}_{2}=\mathrm{J}_{0}\right], \mathrm{J}_{0}$ étant l'intervalle de réalisation du processus énonciatif, on a dans REN : il-est-faux $\left(\operatorname{PROC}_{\mathrm{J} 0}(\mathrm{q})\right)$; on en déduit alors dans REN que : $\underline{\text { il-est- }}$ $\underline{\operatorname{faux}}\left(\operatorname{PROC}_{\mathrm{J} 0}(\mathrm{p})\right)$.

${ }^{20} \mathrm{La}$ formalisation revient à : $1^{\circ}$ ) poser dans RPOS l'équivalence 'ETAT $\mathrm{O}_{\mathrm{O} 1}(\mathrm{p}) \Leftrightarrow \operatorname{PROC}_{\mathrm{J} 2}(\mathrm{q})$ ' avec $\left[\mathrm{J}_{2} \subseteq \mathrm{O}_{1}\right]$ (ou encore $\left[\mathrm{J}_{2}>\mathrm{O}_{1}\right]$ ) $; 2^{\circ}$ ) par synchronisation entre les référentiels RPOS et REN, on vérifie que, dans REN, on a (ou on n'a pas) la réalisation suivante : est-vrai $\left(\operatorname{ETAT}_{\mathrm{O} 1}(\mathrm{p})\right)$ avec $\left[\delta\left(\mathrm{O}_{1}\right)=\delta\left(\mathrm{J}_{0}\right)\right]$, où $\mathrm{J}_{0}$ est l'intervalle de réalisation du processus énonciatif; dans le cas positif, on a : est-vrai $\left(\mathrm{PROC}_{\mathrm{J} 2}(\mathrm{q})\right)$ avec $\left[\delta\left(\mathrm{J}_{2}\right)=\delta\left(\mathrm{J}_{0}\right)\right]\left(\right.$ ou encore $\left.\left[\delta\left(\mathrm{J}_{2}\right)>\delta\left(\mathrm{J}_{0}\right)\right]\right)$ dans REN.

${ }^{21} \mathrm{La}$ formalisation revient à : $1^{\circ}$ ) poser dans le référentiel RPOS l'équivalence ' $\mathrm{ETAT}_{\mathrm{O} 1}(\mathrm{p}) \Leftrightarrow \operatorname{PROC}_{\mathrm{J} 2}(\mathrm{q})$ ' avec [J2 $\subseteq \mathrm{O} 1] ; 2^{\circ}$ ) par synchronisation, on peut confronter l'état posé préalablement comme simplement possible dans RPOS avec l'état effectivement réalisé dans REN, or est-faux $\left(\mathrm{ETAT}_{\mathrm{Ol}}(\mathrm{p})\right)$ avec $\left[\delta\left(\mathrm{O}_{1}\right)=\delta\left(\mathrm{J}_{0}\right)\right]$, $\mathrm{J}_{0}$ étant l'intervalle de réalisation du processus énonciatif ; il s'ensuit alors que on a, dans REN : est-faux $\left(\operatorname{PROC}_{\mathrm{J} 2}(\mathrm{q})\right)$, avec $\left[\delta\left(\mathrm{J}_{2}\right)=\delta\left(\mathrm{J}_{0}\right)\right]$.

${ }^{22}$ Le raisonnement effectué est le suivant: l'état $\mathrm{ETAT}_{\mathrm{O} 1}(\mathrm{p})$ (il avait de l'argent) est posé dans RPOS comme concomitant avec l'événement $\operatorname{EVENT}_{\mathrm{F} 2}(\mathrm{q})$ ( il t'a donné de l'argent) avec $\left[\mathrm{F}_{2} \subseteq \mathrm{O}_{1}\right]$; or, comme on sait par ailleurs, que l'état $\operatorname{ETAT}_{\mathrm{O} 1}(\mathrm{p})$ ne s'est pas réalisé dans le passé du REN, l'état résultant de l'événement $\operatorname{EVENT}_{\mathrm{F} 2}(\mathrm{q})$, par conséquent, n'a pas pu se réaliser (il ne t'a pas donné de l'argent ).
}

${ }^{23}$ Dans RPOS, nous avons l'implication suivante ' $\operatorname{ETAT}_{\mathrm{O} 1}(\mathrm{p}) \Rightarrow \operatorname{EVEN}_{\mathrm{F} 2}(\mathrm{q})$ ', avec $\left[\mathrm{F}_{2} \subseteq \mathrm{O}_{1}\right]$; or, dans REN, on sait que dans le passé on avait: il-est-faux $\left(\operatorname{EVENT}_{\mathrm{F} 3}(\mathrm{q})\right)$, avec $\left[\mathrm{F}_{3}<\mathrm{J}_{0}\right]$, où $\mathrm{J}_{0}$ est l'intervalle de réalisation du processus énonciatif; on en déduit que le processus $\operatorname{PROC}_{\mathrm{J} 3}(\mathrm{q})$, avec $\left[\mathrm{J}_{3} \subseteq \mathrm{F}_{3}\right]$, qui est constitutif de l'événement $\operatorname{EVENT}_{\mathrm{F} 3}(\mathrm{q})$, n'a pas pu avoir lieu également; à la suite d'une synchronisation entre RPOS et REN, qui projette $\mathrm{O}_{1}$ dans le passé avec $\left[\mathrm{J}_{3} \subseteq \mathrm{F}_{3}=\mathrm{F}_{2} \subseteq \mathrm{O}_{1}<\mathrm{J}_{0}\right]$, et à partir de la contraposition dans REN : il-est-faux $\left(\operatorname{PROC}_{\mathrm{J} 3}(\mathrm{q})\right)=>\underline{\text { il- }}$ $\underline{\text { est-faux }}\left(\operatorname{ETAT}_{\mathrm{Ol}}(\mathrm{p})\right)$ nous obtenons, par modus ponens, le détachement: $\underline{\text { il-est-faux }}\left(\operatorname{ETAT}_{\mathrm{O} 1}(\mathrm{p})\right)$ avec $\left[\mathrm{O}_{1}<\mathrm{J}_{0}\right]$. 
Ainsi, l'absence de réalisation de l'état passé $\operatorname{ETAT}_{\mathrm{O} 1}(\mathrm{p})$ devient une explication pour l'absence de la réalisation du processus $\operatorname{PROC}_{\mathrm{J} 3}(\mathrm{q})$ dans REN, et, parallèlement, pour l'absence de l'état résultant de $\operatorname{EVEN}_{\mathrm{F} 3}(\mathrm{p})$.

${ }^{24}$ Dans le RPOS, on a l'implication ' $\operatorname{ETAT}_{01}(\mathrm{p}) \Rightarrow \operatorname{EVENT}_{\mathrm{F} 2}(\mathrm{q})$ ' avec $\left[\mathrm{F}_{2} \subseteq \mathrm{O}_{1}\right]$; or, dans le REN, on sait que l'on a pas eu l'occurrence de l'événement $\operatorname{EVENT}_{\mathrm{F} 3}(\mathrm{q})$, ce qui revient à dire : il-est-faux $\left(\operatorname{EVEN}_{\mathrm{F} 3}(\mathrm{q})\right)$, avec $\left[\mathrm{F}_{3}<\mathrm{J}_{0}\right]$, où $\mathrm{J}_{0}$ est l'intervalle de réalisation du processus énonciatif. En synchronisant le référentiel RPOS avec REN, avec l'identification entre intervalles $\left[\mathrm{F}_{2}=\mathrm{F}_{3} \subseteq \mathrm{O}_{1}<\mathrm{J}_{0}\right]$, par contraposition de l'implication et modus ponens, on obtient finalement : il-est-faux $\left(\operatorname{ETAT}_{01}(\mathrm{p})\right)$ avec $\left[\mathrm{O}_{1}<\mathrm{J}_{0}\right]$.

${ }^{25}$ Dans ce cadre théorique, une analyse systématique des valeurs du conditionnel en français viendrait illustrer la pertinence et l'importance de la prise en compte de plusieurs référentiels (thèse en cours d'Agnès Provôt, Université Paris-Sorbonne).

${ }^{26}$ La valeur de ce que nous appelons «nouvel état» (Desclés 2003) et qui reçoit différentes dénominations dans la littérature (imparfait narratif, imparfait d'ouverture ou de fermeture, imparfait aoristique...), n'est pas une valeur événementielle. En effet, cherchons à opposer, dans le célèbre exemple emprunté à G. Guillaume Cinq minutes plus tard, le train déraillait à Cinq minutes plus tard, le train dérailla (ou a déraillé). Avec le passé simple ou le passé composé, il s'agit de l'énonciation d'une occurrence d'un événement qui s'inscrit dans une structure de succession narrative, c'est donc un événement qui suit d'autres événements et en précède d'autres. L'imparfait indique bien l'occurrence d'un événement mais il focalise l'attention sur ses conséquences, c'est-à-dire sur le «nouvel état» de l'univers narratif, créé par cette occurrence événementielle, d'où ses emplois à la fin d'un paragraphe ou au début pour marquer une véritable rupture par rapport à ce qui précédait : Cette année là, le jeune Louis XIV montait sur le trône : l'état, c'était, désormais, lui, le roi... 